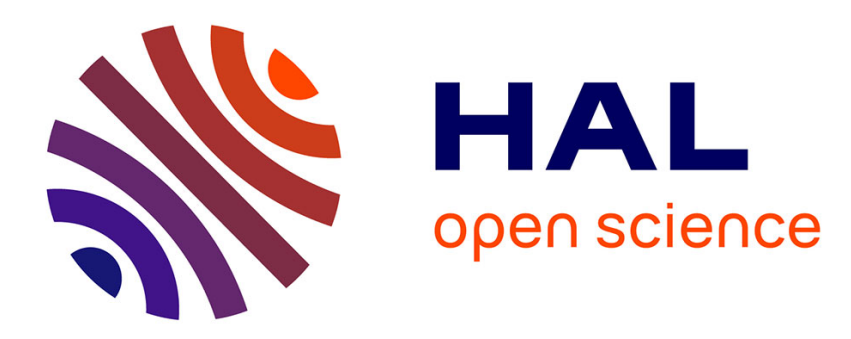

\title{
Elastic and plastic deformations of charge density waves
}

Denis Feinberg, J. Friedel

\section{To cite this version:}

Denis Feinberg, J. Friedel. Elastic and plastic deformations of charge density waves. Journal de Physique, 1988, 49 (3), pp.485-496. 10.1051/jphys:01988004903048500 . jpa-00210720

\section{HAL Id: jpa-00210720 https://hal.science/jpa-00210720}

Submitted on 1 Jan 1988

HAL is a multi-disciplinary open access archive for the deposit and dissemination of scientific research documents, whether they are published or not. The documents may come from teaching and research institutions in France or abroad, or from public or private research centers.
L'archive ouverte pluridisciplinaire HAL, est destinée au dépôt et à la diffusion de documents scientifiques de niveau recherche, publiés ou non, émanant des établissements d'enseignement et de recherche français ou étrangers, des laboratoires publics ou privés. 
Classification

Physics Abstracts

$61.70-62.20-71.45$

\title{
Elastic and plastic deformations of charge density waves
}

\author{
D. Feinberg $(*)$ and J. Friedel $(* *)$ \\ (*) Centre National de la Recherche Scientifique, Laboratoire d'Etudes des Propriétés Electroniques des \\ Solides, associated with Université Scientifique, Technologique et Médicale de Grenoble, B.P. 166, 38042 \\ Grenoble Cedex, France \\ $\left.{ }^{* *}\right)$ Laboratoire de Physique des Solides, associé au CNRS, Université Paris-Sud, Centre d'Orsay, Bât. 510, \\ 91405 Orsay Cedex, France
}

(Reçu le 23 septembre 1987, accepté le 17 novembre 1987)

\begin{abstract}
Résumé. - Nous présentons une théorie élastique des déformations d'une O.D.C. sous l'effet d'un champ électrique, à l'aide d'un simple hamiltonien de phase. L'accent est mis sur la rigidité interne de la modulation qui ressemble en réalité à un «solide électronique », et sur l'anisotropie de ses constantes élastiques. Les déformations continues de l'O.D.C. sont décrites par un tenseur des déformations donné par les variations relatives du vecteur d'onde de la modulation. Les contraintes sont dues aux effets combinés du champ électrique externe et des points d'ancrage. Sous faible contrainte le condensat, globalement accroché, conserve la continuité de phase : la structure n'a pas de défauts. Sous de plus fortes contraintes des dislocations sont nucléées, mettant en jeu une variation locale d'amplitude de l'O.D.C. Nous décrivons le processus de nucléation pour des boucles élémentaires sous champ externe et comparons en conséquence les seuils pour le désancrage de l'O.D.C. des impuretés et des bords (surfaces et interfaces). Une longueur caractéristique est obtenue en dessous de laquelle l'ancrage aux contacts domine et une déformation cohérente (polarisation) apparaît.
\end{abstract}

\begin{abstract}
An elastic theory of CDW deformations under electric fields is presented, on the basis of a simple phase Hamiltonian. The emphasis is put on the internal rigidity of the modulation which indeed resembles an « electronic solid », and on the anisotropy of its elastic constants. CDW's continous deformations are described by a strain tensor given by the relative variations of the wavevector of the modulation. Stresses occur due to the combined effect of external electric field and pinning points. Under low stresses the globally pinned condensate retains phase continuity : the structure has no defects. Under larger stresses dislocation loops are nucleated involving local amplitude variations of the $\mathrm{CDW}$. We describe the nucleation process for elementary loops under an external field and compare as a consequence thresholds for impurity depinning and boundary depinning (surfaces or interfaces). A chăracteristic sample length is found under which contact pinning prevails and a coherent deformation (polarization) is built.
\end{abstract}

\section{Introduction.}

Since the first discovery of collective electronic conduction by sliding charge density waves (CDW) in 1976 [1], an important sum of work has been devoted to the understanding of these very unusual properties [2]. Different classes of compounds, all quasi-unidimensional, share at this time the property of sliding CDW's : tri and tetrachalcogenides as $\mathrm{NbSe}_{3}, \mathrm{TaS}_{3},\left(\mathrm{TaSe}_{4}\right)_{2} \mathrm{I}$ and $\left(\mathrm{NbSe}_{4}\right)_{10 / 3} \mathrm{I}$, molybdenum bronzes $\mathrm{A}_{0.3} \mathrm{MoO}_{3}(\mathrm{~A}=\mathrm{K}, \mathrm{Rb}, \mathrm{Tl})$ and more recently TTF-TCNQ [2, 3]. Of all these compounds only $\mathrm{NbSe}_{3}$ remains metallic at low

\footnotetext{
JOURNAL DE PHYSIQUE. - T. 49, N 3, MARS 1988
}

temperature, the others becoming semiconductors with a Peierls gap opening on the nearly perfectly nested Fermi surface.

The clearest evidence for sliding of the CDW the so-called Fröhlich mode - comes from the observation of periodic "noise " generated by the motion over the underlying periodic pinning potential $[1,2]$. Pinning of the condensate by random impurities is most probably the cause of the finite threshold field for CDW motion and of the strong metastability effects $[4,5]$. Deformations of the CDW have been proven to exist, especially under electric fields [6-9]. Furthermore NMR experiments 
carried on under electric fields on $\mathrm{NbSe}_{3}$ [10] and $\mathrm{Rb}_{0.3} \mathrm{MoO}_{3}$ [11] seem to give clear evidence of a coherent motion of the whole condensate. Unfortunately, even if the basic physics are understood and reasonable theoretical models exist (Ref. [2a] Vol. I), up to now no synthetic picture is able to account for the rich and complex experimental situation.

The quantum origin of CDW formation is well understood : the Peierls instability [12] of the quasi one-dimensional Fermi surface at $q=2 k_{\mathrm{F}}$ induces the condensation of electron-hole pairs at $q$ and $-q$, resulting in a modulation in real space of both the atomic positions and the electronic density, while a gap $2 \Delta$ opens at the Fermi level $\varepsilon_{\mathrm{F}}$. The question of deciding whether CDW motion results of macroscopic tunnelling of the condensate through impurities [13] or from a purely classical process [14, $15]$ is still controversial. In the first eventuality, apparently confirmed by interference experiments [16], these systems would be amongst the few known examples of macroscopic quantum phenomena. On the other hand, it is difficult to ascribe all the observed phenomena to such an effect. On the contrary, low time scale metastability properties are clearly the sign of large length scale relaxation, hardily described but by deformations of a classical medium. We shall restrict ourselves to such a classical approach and do not attempt to cover the eventual crossover to quantum effects.

The lattice modulation and ensuing CDW can be considered as a purely sinusoïdal wave in a continuum. In this approximation the electric charge density along the chains is

$$
\rho(\mathbf{r})=\bar{\rho}[1+A(\mathbf{r}) \cos (q x+\varphi(\mathbf{r}))]
$$

where

$$
q=\frac{2 \pi}{\lambda}, \quad \bar{\rho}=n_{\mathrm{s}} e
$$

and

$$
A(\mathbf{r})=\frac{\Delta(\mathbf{r})}{\varepsilon_{\mathrm{F}}} \operatorname{Ln} \frac{\varepsilon_{\mathrm{F}}}{\Delta(\mathbf{r})} .
$$

The CDW is locally described by its amplitude or by the gap $\Delta$, and by its phase $\varphi$ respective to the fixed lattice. It can be alternatively characterized by a complex two-component order parameter $\psi=\left(\Delta / \Delta_{0}\right) \mathrm{e}^{i \phi}$ where $\Delta_{0}$ is the $T=0$ gap for an undistorted $\mathrm{CDW}$. As a consequence, such a system has to be compared to other systems described by two-component order parameters such as superfluids and superconductors, $x-y$ magnets or some liquid crystals.

The Bose condensation of electron-hole pairs at $\pm q, q=2 k_{\mathrm{F}}[17,18]$ gives rise to a coherence length $\xi$, indeed very similar to the BCS coherence length and measuring the order of magnitude of the size of the electron-hole pair. In the chain direction it is given by $\xi_{\|} \sim \hbar v_{\mathrm{F}} / \pi \Delta$ where $v_{\mathrm{F}}$ is the Fermi velocity. It can be much smaller in the transverse direction, being due to the weak interchain couplings. $k_{\mathrm{F}}^{-1}$ being of the same order of magnitude as the parallel unit length $a_{\|}$, one sees that $\xi_{\|} / a_{\|} \sim \varepsilon_{\mathrm{F}} / \Delta$ which is of the order of several tenth, giving $\xi_{\|} \sim 100 \AA$ as an order of magnitude. This shows that CDW electrons keep their itinerant character and are not truly localized by their effective interaction. An alternative model due to Aubry [19] considers the opposite case of a "polaronic crystal » valid in strong enough coupling. An heuristic argument can be given to explain how real space electronic ordering can originate from a condensation of « large » electron-hole pairs in weak coupling. One must take into account the fact that the effective mass for Fröhlich electrons coupled to heavy ions is the Fröhlich mass $M \sim 10^{2}$ to $10^{3} \mathrm{~m}_{\mathrm{e}}$, thus the zero-point kinetic energy of the modulation is of order $\hbar^{2} k_{\mathrm{F}}^{2} / 2 M$, smaller than the interaction $\Delta$ which stabilizes the modulation. Thus the first of the two inequalities

$$
\frac{\hbar^{2} k_{\mathrm{F}}^{2}}{2 M}<\Delta<\varepsilon_{\mathrm{F}}
$$

expresses the conditions for a quantum «electron solid » to form, while the second one expresses that amplitude variations of the CDW can occur only on distances longer than the coherence length $\xi$.

We want to point out that the rigidity of CDW's, as stressed by Anderson [4, 20] is an essential feature which has not received enough attention up to now. As a consequence one can hope to build an elastic description of CDW deformation and motion, similar to that of a three-dimensional solid. A few conditions must be put forward to justify such a picture. First, it is valid only at low temperature, far enough from the Peierls transition. The CDW actually softens close to $T_{\mathrm{p}}$ due to the decrease of $\Delta$ and the first inequality of (1.4) might not be fulfilled anymore. Secondly, the following description assumes that all long-range Coulomb interactions internal to the CDW degrees of freedom are screened by normal carriers, still existing in the ground state (case of $\mathrm{NbSe}_{3}$ ) or thermally excited above the gap in the semiconducting low temperature state of other compounds as o- $\mathrm{TaS}_{3}$ or the «blue bronze » $\mathrm{A}_{0.3} \mathrm{MoO}_{3}(\mathrm{~A}=\mathrm{K}$, $\mathrm{Rb})$. Such a picture breaks down at very low temperatures in the insulating phase.

Within such a phenomenological frame a CDW can be viewed as a classical solid, pinned by random impurities and crystal boundaries. Applying an electric field smaller than the critical field $E_{T}$, its reacts 
as an ordinary crystal reacts to an external mechanical uniaxial stress, causing strain and stress fields within the sample. Simple arguments can be given to describe the elastic response of the system and the approach to depinning. A full description of depinning phenomena will not be attempted here. Nevertheless, the fact that a CDW encounters a finite global pinning force, i.e. an infinite viscosity for $E<E_{T}$ is by itself a proof of its solid friction on the underlying lattice. No hydrodynamical approach based on short-range forces rather than long-range elastic forces is able to account for such an observation.

Moreover, the present picture allows to attempt a description of topological defects occurring in the condensate. Very similar to vortex loops or flux lines in superfluids and type II superconductors respectively, they are also very close to cristalline dislocations. They break the phase continuity and form under strong enough internal strains, while low strain only break the long range coherence of the phase. As will be shown, such CDW defects play an important role in CDW depinning and motion.

Only incommensurate CDW's will be considered here. Some effects of commensurability have been discussed in previous works [21, 22] and together with a review of CDW defects in blue bronzes [23].

The long-range character of elastic forces within the $\mathrm{CDW}$ has a direct consequence : finite-size effects play a crucial role in CDW pinning and depinning. Actually, speaking of local pinning forces is meaningless, on the contrary the elastic strains to be overcome by the external electric force are themselves a result of the overall and macroscopic distortion of the CDW's « crystal ». A direct consequence for contact pinning, as will be shown in section 4 is that applied stresses vary linearily with the sample length $L$, thus depinning fields vary roughly as $L^{-1}$. Thus short samples are dominated by contact pinning and long samples by bulk pinning. A more accurate evaluation of threshold fields can be obtained by comparing the applied stress with the critical stress required to break locally the condensate. A simple explanation of some general features of contact and surface pinning will be given in section 4 , together with orders of magnitude estimates. We also emphasize the crucial role of the anisotropy of the CDW elastic constants which dramatically decreases the thresholds for dislocation nucleation.

The plan of this article is as follows. In section 2 we recall the basic Hamiltonians for phase and amplitude deformation of an incommensurate CDW. CDW topological defects are reviewed in section 3 together with a discussion of their nucleation mechanisms. Section 4 is devoted to an examination of finite size effects, i.e. pinning and depinning from surfaces and interfaces (contacts).

\section{Elastic deformations of charge density waves.}

The starting point of our analysis is the McMillanLee-Rice free energy which, for the isolated CDW in three dimensions, is given by $[24,25]$

$$
\begin{array}{r}
F=F_{0} \int \mathrm{dr}\left[-t|\psi|^{2}+\frac{1}{2}|\psi|^{4}+\xi_{x}^{2}\left(\frac{\partial \psi}{\partial x}\right)^{2}+\right. \\
\left.+\xi_{y}^{2}\left(\frac{\partial \psi}{\partial y}\right)^{2}+\xi_{z}^{2}\left(\frac{\partial \psi}{\partial z}\right)^{2}\right]
\end{array}
$$

where $t=-\left(T-T_{\mathrm{c}}\right) / T_{\mathrm{c}}$ and $\xi_{x}, \xi_{y}, \quad \xi_{z}$ are the coherence lengths, $x$ being along the chain axis (in difference with notations of reference [25] $\xi_{x}=$ $\hbar v_{\mathrm{F}} / \pi \Delta_{0}$ is the BCS-like coherence length). (2.1) is actually a Ginzburg-Landau expansion the elastic part of which can be derived in one dimension from microscopical grounds [26]. $F_{0}$ is thus given by

$$
F_{0}=\frac{\Delta_{0}^{2}}{\varepsilon_{\mathrm{F}} \Omega} \frac{\pi^{2}}{2} \frac{a_{x}}{\lambda}
$$

where $a_{x}, a_{y}, a_{z}$ are the unit lengths and $\Omega=a_{x} a_{y} a_{z}$ the unit volume, $\lambda$ the CDW wavelength. On the other hand, $\xi_{y}$ and $\xi_{z}$ depending on the nature and strength of interchain couplings are purely phenomenological parameters and can be much smaller than $\xi_{x}$. We shall not try to go beyond the continuous picture, even if the limiting case $\xi_{y} \sim a_{y}$, $\xi_{z} \sim a_{z}$ could be reached in the most anisotropic compounds such as blue bronzes.

Moreover (2.1) assumes short-range interactions of the condensate with itself and should be modified in semiconducting compounds at low temperatures $[27,28]$.

The scaled form of (2.1) obtained by setting $\tilde{x}=x, \quad \tilde{y}=y\left(\xi_{x} / \xi_{y}\right), \quad \tilde{z}=z\left(\xi_{x} / \xi_{z}\right) \quad$ is $\quad$ (with $\tilde{\nabla}=\mathrm{d} / \mathrm{d} \tilde{r})[25]$

$$
\begin{aligned}
F= & F_{0}\left(\xi_{y} \xi_{z} / \xi_{x}^{2}\right) \times \\
& \times \int \mathrm{d} \mathbf{r}\left[-t|\psi|^{2}+\frac{1}{2}|\psi|^{4}+\xi_{x}^{2}|\tilde{\nabla} \psi|^{2}\right] .
\end{aligned}
$$

It looks formally very similar to the GinzburgLandau free energy of superfluid $\mathrm{He}^{4}$ [29]. Nevertheless the two systems are very different in the sense that in CDW's $\nabla \varphi$ is associated to a static deformation in space (like that of an elastic crystal) and not to a real particle current.

The relevant variable to be coupled to external forces is the phase variable, whose variations induce in turn amplitude variations. Neglecting the last ones one recovers the usual phase elastic energy

$$
\begin{aligned}
F_{\mathrm{el}} & =\int \mathrm{d} \tilde{\mathbf{r}} \frac{1}{2} K|\psi|^{2}|\tilde{\nabla} \varphi|^{2} \\
K & =\frac{\hbar v_{\mathrm{F}}}{2 \pi a_{y} a_{z}} \cdot \frac{\xi_{y} \xi_{z}}{\xi_{x}^{2}} .
\end{aligned}
$$


In other terms the CDW has three elastic constants : $K_{x}=\hbar v_{\mathrm{F}} / 2 \pi a_{y} a_{z}, K_{y}=K_{x}\left(\xi_{y} / \xi_{x}\right)^{2}, K_{z}=$ $K_{x}\left(\xi_{z} / \xi_{x}\right)^{2}$. Nevertheless (1.1) shows that the CDW's deformations are determined by a scalar field $\varphi(x, y, z)$ describing uniaxial distortions (along $x)$. The local displacement vector is $\mathbf{u}=-(\varphi / q) \mathbf{x}$ and allows to define the non-zero strain tensor components :

$$
\begin{gathered}
e_{x x}=-\frac{1}{q} \frac{\partial \varphi}{\partial x}, \quad e_{x y}=e_{y x}=-\frac{1}{2 q} \frac{\partial \varphi}{\partial y}, \\
e_{x z}=e_{z x}=-\frac{1}{2 q} \frac{\partial \varphi}{\partial z} .
\end{gathered}
$$

Long wavelength variations of the phase can be understood as local modifications of the CDW wave vector, i.e. $\partial \varphi / \partial x=\delta q_{x}, \nabla_{\perp} \varphi=\delta q_{\perp}$. The first type of distortions are compressions or dilatations and create an uncompensated charge through the variation of the Fermi vector $k_{\mathrm{F}}=(1 / 2) q$, whose average is given by

$$
\rho_{\mathrm{s}}=\frac{n_{\mathrm{s}} e}{q} \frac{\partial \varphi}{\partial x}=-\left(n_{\mathrm{s}} e\right) e_{x x} .
$$

The second type of distortions are shear-like, bear no charge and correspond to rotating locally the CDW's wave vector (Fig. 1). The elapstic energy (2.4), assuming $|\psi|=1$, can be cast into the usual form $F_{\mathrm{el}}=\int \mathrm{d} \mathbf{r}(1 / 2)[\sigma][e]$ by defining the stress

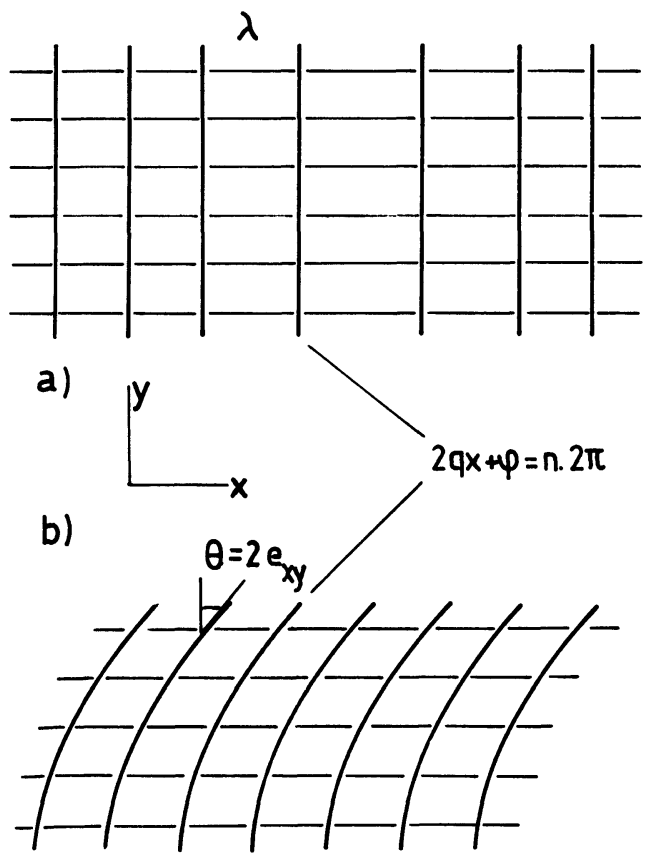

Fig. 1. - The two types of deformations of CDW's : a) compression (or dilatation) involves a longitudinal strain $\left.e_{x x} ; \mathrm{b}\right)$ shear involves a strain $e_{x \perp}\left(=e_{x y}, e_{x z}\right)$. tensor

$$
\begin{gathered}
\sigma_{x x}=q^{2} K_{x} e_{x x}, \quad \sigma_{x y}=q^{2} K_{y} e_{x y}, \\
\sigma_{x z}=q^{2} K_{z} e_{x z} .
\end{gathered}
$$

Coupling the CDW to an external force $F(\mathbf{r})$ due to impurities, boundaries or electric field, only the $x$ component produces a work and one gets the equilibrium condition

$$
\sum_{i} \frac{\partial \sigma_{i x}}{\partial i}+F_{x}=0, \quad i=x, y, z
$$

where $F_{x}$ is a volume force, thus

$$
K \tilde{\nabla}^{2} \varphi+\frac{F_{x}(\mathbf{r})}{q}=0 .
$$

In case of an applied electric field $E$, the coupling energy is

$$
U_{\mathrm{f}}=-\int \mathrm{d} \mathbf{r} \frac{n_{\mathrm{s}} e}{q} \varphi E
$$

thus (2.9) reads

$$
K_{x} \tilde{\nabla}^{2} \varphi+\frac{n_{\mathrm{s}} e E}{q}=0 .
$$

Pinning at low fields requires introducing the pinning energy [24]

$$
\begin{aligned}
& F_{\text {pin }}=-\int \mathrm{d} \mathbf{r} \rho_{1}|\psi| \sum_{i} V\left(\mathbf{r}-\mathbf{r}_{i}\right) \times \\
& \times \cos \left(\varphi-\varphi_{i}\right)+F_{\text {pin }}^{b}
\end{aligned}
$$

where $\varphi_{i}=-q X_{i}$ is the imposed phase at site $r_{i}, \rho_{1}=\bar{\rho} A$ (Eq. 1.1) and $F_{\text {pin }}^{b}$ is a similar term expressing strong pinning at surfaces and interfaces (see Sect. 4). $V$ is a short-range impurity potential.

In strong pinning the phase is blocked at site $i$ and on the boundaries of the system so (2.11) appears to be a Poisson equation with Dirichlet boundary conditions which can be solved exactly only in a few simple configurations, as we show later.

This picture neglects the variation of the amplitude at strong pinning points, due to a local variation of the order parameter $|\psi|$ [24]. Nevertheless the expected effect is a slight modification of the elastic constant (Eq. 2.4), localized in a coherence volume around an impurity (see the Appendix). It is thus reasonable to solve the simplified equation (2.11) to obtain the phase configuration, at least in low fields. Furthermore, it should be noted that boundary conditions imposing phase near impurities have to be applied on a small surface surrounding the impurity, the radius of which depends on the strength of the impurity. This fixes a small length scale which will be very critical as far as the local strains due to strong pinning are concerned. 


\section{Defects in charge density waves.}

3.1 CDW DisLOCATIONS. - Let us now discuss topological defects of incommensurate CDW's, i.e. inducing singularities of the phase field. Such defects are characterized by the dimension of space and of the order parameter (here, two) thus are very similar to vortices (in $d=2$ ) and vortex lines in $X-Y$ magnets and superfluids. Their physical properties are closer to that of dislocation lines in crystals, with the simplication quoted above of uniaxial displacements of the CDW « lattice ». The existence of such dislocations have been proposed by Lee and Rice [25]. The Burgers vector of perfect dislocations is defined by [21] :

$$
\mathbf{B}=\oint_{C} \nabla \mathbf{u} \mathrm{d} \mathbf{r}=-\frac{\mathbf{x}}{q} \oint_{C} \nabla \varphi \mathrm{d} \mathbf{r}
$$

where $\mathrm{C}$ is a circuit enclosing the dislocation line. Because of the scalar nature of the displacement field, edge dislocations (orthogonal to the chains) and screw dislocations (parallel to the chains) have the same topology $\varphi=\theta$ where $\theta$ is the angle in scaled coordinates in the plane normal to the dislocation line. Thus for an edge dislocation parallel to $\mathbf{z}$ the stress field at point $\mathbf{r}=(x, y, 0)$ from the dislocation is :

$$
\begin{aligned}
\sigma_{x x} & =q K_{x} \frac{\alpha_{y} y}{x^{2}+\left(\alpha_{y} y\right)^{2}} \\
\sigma_{x y} & =-\frac{q K_{x}}{2 \alpha_{y}} \frac{x}{x^{2}+\left(\alpha_{y} y\right)^{2}} \\
\alpha_{y} & =\frac{\xi_{x}}{\xi_{y}} ; \quad \alpha_{z}=\frac{\xi_{x}}{\xi_{z}}
\end{aligned}
$$

One gets similar expressions for a line parallel to $y$, by permuting $y$ and $z$. For a screw line, on the other hand,

$$
\begin{aligned}
& \sigma_{x y}=\frac{q K_{x}}{2 \alpha_{y} \alpha_{z}} \frac{z}{z^{2}+\left(\frac{\alpha_{y}}{\alpha_{z}}\right)^{2} y^{2}} ; \\
& \sigma_{x z}=-\frac{q K_{x}}{2 \alpha_{y} \alpha_{z}} \frac{y}{y^{2}+\left(\frac{\alpha_{z}}{\alpha_{y}}\right)^{2} z^{2}} .
\end{aligned}
$$

The core properties of these dislocations is similar to that of vortex or flux lines in superfluid $\mathrm{He}^{4}$ or type II superconductors respectively. Here the core will be anisotropic and extend over a coherence area of size $\xi$ in each direction. The gap goes to zero in the middle of the core, which should be more nearly metallic.

A strong anisotropy has dramatic consequences on the dislocation line energy. Per unit length of screw dislocation one gets [25]

$$
E_{\mathrm{s}}=\pi \sqrt{K_{y} K_{z}} \operatorname{Ln}\left(\frac{\tilde{R}}{\tilde{\xi}}\right)
$$

where $\tilde{R}$ is a scaled large-distance cut-off and $\tilde{\xi}$ a short-distance one close to the coherence length and modified in order to account for the amplitude deformation at the core. One must underline that, like in superfluid systems, such corrections should not be more than a fraction of the elastic part (the phase field energy), just because the gap falls gradually to zero inside the core region.

One has similarly for an edge dislocation parallel to $\mathbf{y}$

$$
E_{\mathrm{e}}=\pi \sqrt{K_{x} K_{z}} \operatorname{Ln}\left(\frac{\tilde{R}}{\tilde{\xi}}\right) .
$$

Thus screw dislocations cost less energy, by a factor $\xi_{\perp} / \xi_{x}$ (if $\xi_{y} \sim \xi_{z} \sim \xi_{\perp}$ ). The effect is more dramatic if one calculates the energy of the smallest possible loop, which is the relevant energy for nucleation events. Edge loops of size $\xi_{\perp}$ (in the $y z$ plane) and mixed loops of dimension $\xi_{x}$ times $\xi_{\perp}$ in a plane containing $x$ (Fig. 2) have roughly the same energy, of order

$$
E_{0}^{\text {loop }} \simeq 4 \pi K_{x} \frac{\xi_{\perp}^{2}}{\xi_{x}} \simeq \Delta_{0}\left(\frac{\xi_{\perp}}{a_{\perp}}\right)^{2}
$$

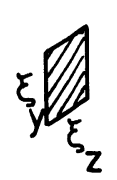

a)

)

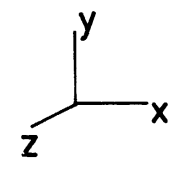

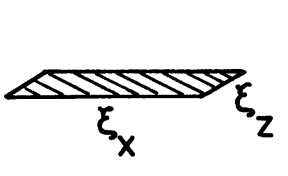

b)
Fig. 2. - Smallest dislocation loops a) edge-like (e) ; b) mixed loop (e and s).

(setting $\tilde{R} \sim \tilde{\xi}$ in (3.4) and (3.5)). Therefore an anisotropy of 10 in the coherence length leads to a reduction factor of 100 in the loop energy compared to an isotropic system. This could be enough to allow thermal activation of such loops, an eventuality considered by Maki [30]. Actually with $\xi_{\perp} \sim a_{\perp}$ the nucleation energy is of order $\Delta_{0}$, thus a few hundredths of electron volts.

Apart from strongly reducing the net energy of dislocations, especially screw one which involve only shear, the anisotropy of dislocation cores has also consequences on their dynamics : edge dislocations with narrow cores along transverse directions encounter a lattice friction for climb due to PeierlsNabarro barriers between chains. These effects have 
been qualitatively discussed elsewhere $[22,23]$ and can strongly inhibit the climb motion of edge dislocations involved at contacts by the motion of CDW's [31]. This effect is extremely sensitive to the actual value of $\xi_{\perp} / a_{\perp}$, for the height of the PeierlsNabarro barrier should vary roughly as $\exp \left(-\xi_{\perp} / a_{\perp}\right)[32]$.

More generally, such CDW dislocations can move through the pinned condensate, as stressed by Lee, Rice [25]. One should note that they do not interact directly with the electrical field but rather move under the effect of internal elastic forces, the Peach and Koehler force per unit length being [32]

$$
\mathbf{F}_{\mathrm{PK}}=\lambda \mathbf{x}[\tilde{\boldsymbol{\sigma}}] \times \mathbf{L}
$$

$[\tilde{\sigma}]$ being the scaled stress tensor and $\mathbf{L}$ the unit vector along the line $L, \mathbf{F}_{\mathrm{PK}}$ is normal to $\mathbf{L}$. For instance such forces are responsible for the climb of edge dislocations near contacts. Such an analysis must be completed in the insulating regime where for instance an edge dislocation, due to (2.6) and to the phase topology carries a linear dipole density. One consequence is to increase notably the energy of edge dislocation lines, in difference with screw lines. Such effects should be relevant for a discussion of injection mechanisms at very low temperatures in semiconducting CDW compounds.

Other low-dimensional singularities of the order parameter, with rotational symmetry can be considered, as 'in smectics: disclinations and point singularities. They are stable only under an applied volume force and are solution of equation (2.11).

They have been shown to be less stable than purely elastic deformations, unless they are strongly localized [23].

3.2 NUCLEATION OF CDW DEFECTS. - Let us turn now to the problem of the nucleation of such defects. One has to distinguish, firstly between zero field and finite field configurations, secondly between bulk and boundary effects, thirdly between low and high temperature regimes.

Thermal nucleation of dislocation loops could occur at equilibrium for very anisotropic compounds and at high enough temperatures (see Eq. (3.6)). Blue bronzes and $\mathrm{TaS}_{3}$ close to the transition temperature could be candidates to such a thermal proliferation of defects, a consequence of which would be to destroy quickly the phase coherence thus lowering the transition temperature, by a kind of defect-induced melting process as in the Shockley model [33] for crystals or Feynman's model [34] for superfluids.

A more likely possibility is defect nucleation under internal stresses. These can be due to the effects of incoherent pinning impurities or to an external applied field. In the first case the situation is very similar to that of $X-Y$ magnets in random fields
[35]. In the case of CDW's a rough argument can be given as follows : let us assume a distribution of strong pinning centres with average distance $l$ and density $l=n_{i}^{-1 / d}$ in dimension $d(d=2,3)$. Resolving the strains by an elastic deformation matching random phases $\varphi_{i}$ at different sites gives an average elastic energy of order $\mathrm{Kl}^{-2}=K n_{i}^{2 / d}$ per unit volume, thus $\mathrm{Kn}_{i}^{2 / d-1}$ per impurity. On the other hand, one can keep the phase locally much closer to $\varphi_{i}$ near each impurity and match domains of constant phase by dislocation loops. This needs at most one loop of size $l(d=3)$ or one vortex $(d=2)$ per impurity, thus if $l>\xi$ an energy of order $K l^{d-2} \ln (l / \xi) \sim K n_{i}^{2 / d-1}$ per impurity, same as above. The pinning energy being localized in the immediate vicinity of the impurity is nearly the same in these two extreme situations. Therefore the total energy also scales in the same way and one needs a more accurate analysis to decide whether the " good " picture for strong pinning is that of a phase field continuously distorting or domains matched by dislocation loops (as the $\varphi$ vortices assumed by Maki [30]).

The most interesting source of CDW defects is field-induced strains. The elastic energy stored by the combined effect of external field and pinning allows to overcome the barrier for nucleation. A possible process is the Frank-Read mechanism [32], starting from preexisting dislocation lines, as proposed by Lee and Rice [25]. This is unlikely in CDW compounds for two reasons. First, the Burgers vector having one single orientation, parallel to the chains, no Frank network of dislocation can build up. Secondly, successive loops strongly pinned by impurities in different glide planes would require a somewhat unlikely geometry [23].

Let us now describe an athermal nucleation process in presence of a local elastic stress $\sigma$. Acting as to increase by $\mathrm{d} R$ the radius of a loop of radius $R$, its work is (from (3.7))

$$
\mathrm{d} W=\lambda \tilde{\sigma}(2 \pi \tilde{R} \mathrm{~d} \tilde{R})
$$

$\tilde{\sigma}$ can be either a compression stress $\left(\tilde{\sigma}=\tilde{\sigma}_{x x}\right)$ acting on an edge loop, or a shear stress $\tilde{\sigma}=\tilde{\sigma}_{x y}$, $\tilde{\sigma}_{x z}$ ) acting on a mixed loop lying in a glide plane containing $\mathbf{x}$ (Fig. 3 ).

On the other hand the energy of the loop, given by $2 \pi^{2} \tilde{K} \tilde{R} \operatorname{Ln}(\tilde{R} / \tilde{\xi})$ where $\tilde{\xi}$ has the order of magnitude of the scaled coherence length, varies as

$$
\mathrm{d} E=2 \pi^{2} \tilde{K}\left(\operatorname{Ln} \frac{\tilde{R}}{\tilde{\xi}}+1\right) \mathrm{d} \tilde{R} .
$$

Equating (3.8) and (3.9) leads to

$$
\tilde{R}^{-1}\left(\operatorname{Ln} \frac{\tilde{R}}{\tilde{\xi}}+1\right)=\frac{\lambda \tilde{\sigma}}{\pi \tilde{K}} .
$$


a)

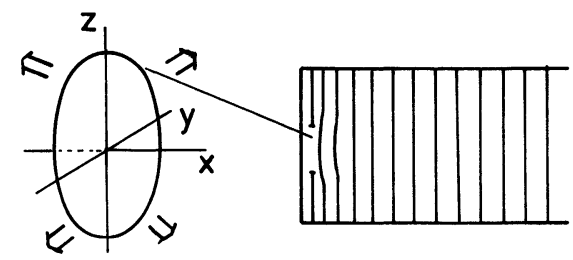

b)

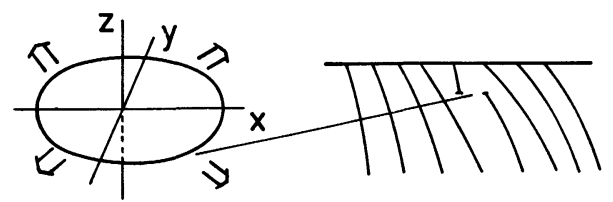

Fig. 3. - Nucleation under external stress of a) an edge loop; b) a mixed loop.

Minimizing expression (3.10) with respect to $\tilde{R}$ gives the minimal stress $\tilde{\sigma}_{\mathrm{c}}$ for $\tilde{R}=\tilde{\xi}$ which is actually the size of the critical loop considered previously (Eq. (3.6)). The critical stress corresponds to a critical strain $\tilde{e}_{\mathrm{c}}=\tilde{\sigma}_{\mathrm{c}} / q^{2} \tilde{K}$, thus given by

$$
\tilde{e}_{\mathrm{c}}=\frac{\lambda}{4 \pi \tilde{\xi}}
$$

or

$$
\begin{aligned}
\frac{\delta q_{\|}}{q} & =e_{x x}^{\mathrm{c}}=\frac{\lambda}{4 \pi \xi_{x}} ; \\
\frac{\delta q_{\perp}}{q} & =e_{x \perp}^{\mathrm{c}}=\frac{\lambda}{4 \pi \xi_{\perp}}
\end{aligned}
$$

respectively for compression and shear $\left(e_{x \perp}=e_{x y}, e_{x z}\right)$.

The above argument is directly inspired from mechanisms involved in crystalline elasticity under a localized mechanical stress. It is very sensitive to imperfections or inhomogeneities which could locally increase the stress, thus facilitate defect nucleation and lower the threshold field. A related argument given by Maki [30] considers on the contrary thermal nucleation for a given stress $\sigma$. The athermal process described above may occur on very strong bulk pinning centres such as grain boundaries or crystalline dislocations, but is much more relevant for contact and surface depinning, as will be described in the next section.

Equation (3.12) provides an order of magnitude of critical relative variations of the wavevector for breaking locally the condensate at low temperatures : $e_{x x}^{\mathrm{c}} \sim 10^{-2}$ and $e_{x \perp}^{\mathrm{c}} \sim 10^{-1}$ for $\xi_{x} \sim 100 \AA$ and $\xi_{\perp} \approx \lambda \approx 10 \AA$.

\section{Finite-size effects.}

Any accurate discussion of depinning mechanisms requires solving properly the equilibrium equation (2.9) where the force $F_{x}$ contains both the electrical force and the periodic pinning force de- duced from (2.12). The problem involving both disorder and nonlinearity is formidable and it is simpler, at least for strong pinning, to assume that pinning centres impose boundary conditions on the linear equation (2.11), to solve the obtained $\mathrm{Di}$ richlet problem and to compare the local stresses deduced from the solution in the vicinity of pinning centres to critical pinning stresses representing the strength of these centres. Two types of critical stresses show out : the first ones assume no breaking of the condensate at depinning. They are directly related to the strength of the impurity potential. If this is of order $V_{i}$ a typical order of magnitude of this local critical «elastic » stress is simply $\sigma_{\mathrm{c}} \sim \rho_{1}|\psi| V_{i}$ as given by equation (2.12). The other critical stresses are those calculated in section 3 for breaking locally the condensate. We shall consider them as "plastic» stresses $\sigma_{\mathrm{c}}^{\mathrm{pl}}$. They are indeed very similar to plastic critical stresses involved in the elastic limit of crystals under mechanical stresses, the so-called yield point of alloys [32].

The estimates obtained in this way obviously neglect the effects of amplitude variations which should not bring qualitative modifications, at least for fields smaller or of the order of depinning fields (see the Appendix). On the contrary, dynamical studies of depinning at very high fields have shown long-range diffusion of amplitude fluctuations [36] an cannot be described by a time-dependent version of an elastic phase analysis.

4.1 ConTACT PINNING. - Let us consider first the situation of contact pinning in a one-dimension configuration (Fig. 4). This would apply for samples short enough so that bulk depinning is obtained before contact depinning. Equation (2.11) then writes

$$
K_{x} \frac{\mathrm{d}^{2} \varphi}{\mathrm{d} x^{2}}+\frac{n_{\mathrm{s}} e E}{q}=0
$$

which, with $\varphi(0)=\varphi(L)=0$ gives

$$
\varphi(x)=\frac{n_{\mathrm{s}} e E}{2 q K_{x}} x(L-x) .
$$
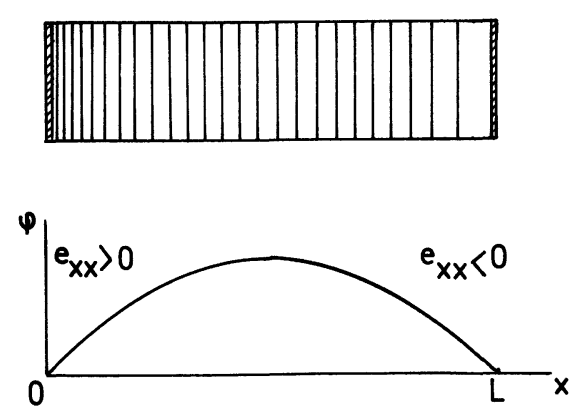

Fig. 4. - Boundary (contact) pinning in a one-dimensional configuration. 
This parabolic profile shows that the phase shift from the $E=0$ value can be very large inside the sample. Actually (4.2) allows to define a fielddependent coherence length on which $\varphi$ varies of $2 \pi$ (see also Eq. (4.13))

$$
\xi_{x}^{\mathrm{f}}=4 \pi \sqrt{\frac{K_{x}}{n_{\mathrm{s}} e E \lambda}} .
$$

The second consequence of (4.2) is the value of the maximum applied strain, obtained at $x=0$ and $x=L$

$$
e_{\operatorname{Max}}=\frac{n_{\mathrm{s}} e E L}{2 q^{2} K_{x}}
$$

This is the relevant quantity to be compared with the critical strains at pinning points $e_{\mathrm{c}}^{\mathrm{el}}=\sigma_{\mathrm{c}}^{\mathrm{el}} / q^{2} K_{x}$ and $e_{\mathrm{c}}^{\mathrm{pl}}=\sigma_{\mathrm{c}}^{\mathrm{pl}} / q^{2} K_{x}$. As a result, the experimental parameter will be the applied voltage $V=E . L$. The critical voltage is obtained by equating (4.4) to (3.11)

$$
V_{T}=\frac{2 \pi K_{x}}{n_{\mathrm{s}} e \xi_{x} \lambda}=\frac{\hbar v_{\mathrm{F}}}{n_{\mathrm{s}} e\left(\lambda a_{y} a_{z}\right) \xi_{x}}
$$

Using (2.7) and (4.4) one can also write the following relation between critical stress and voltage

$$
V_{T}=\frac{2 \sigma}{n_{\mathrm{s}} e} .
$$

As an order of magnitude for blue bronzes, one can take $v_{\mathrm{F}} \sim 10^{8} \mathrm{~cm} . \mathrm{s}^{-1}, n_{\mathrm{s}}=5 \times 10^{21} \mathrm{~cm}^{-3}$ and $\xi_{x} \sim$ $100 \AA, \lambda=4 / 3 a_{x} \sim 10 \AA, a_{y}=16 \AA, a_{z}=10 \AA$ so one finds $V_{T}=8 \mathrm{mV}$. Experimental values of $2.4 \mathrm{mV}$ have been found for $\mathrm{K}_{0.3} \mathrm{MoO}_{3}$ by Arbaoui et al. [37]. Due to the uncertainty of some of the experimental parameters one cannot expect more than an order of magnitude agreement. For orthorhombic $\mathrm{TaS}_{3}$, with same values of $v_{F}$ and $\xi_{x}$ (the gaps of $\mathrm{TaS}_{3}$ and $\mathrm{K}_{0.3} \mathrm{MoO}_{3}$ are comparable) but $n_{\mathrm{s}}=4.5 \times 10^{21} \mathrm{~cm}^{-3}, \quad \lambda=4 a_{x}, \quad a_{x}=3.3 \AA$, $a_{y}=39 \AA, a_{z}=15 \AA$ one gets $V_{T} \sim 2.5 \mathrm{mV}$. Values of $6 \mathrm{mV}$ have been reported by Mihaly et al. [38].

These values can be very sensitive to contact geometry creating locally strain concentrations by distorting the CDW. The effect can be very large in the configuration used for $\mathrm{NbSe}_{3}$ samples where values $V_{T} \sim 0.1 \mathrm{mV}$ have been reported [39] while expression (4.5) leads to $V_{T} \sim 10 \mathrm{mV}$, thus two orders of magnitude higher. Such a discrepancy is probably due to strong CDW deformations induced by the contact geometry (Fig. 5), as shown in the following.

One must distinguish between contact configurations which distort very slightly or strongly the CDW «crystal ». In the first case encountered for « frontal» contacts, deposited uniformly on the

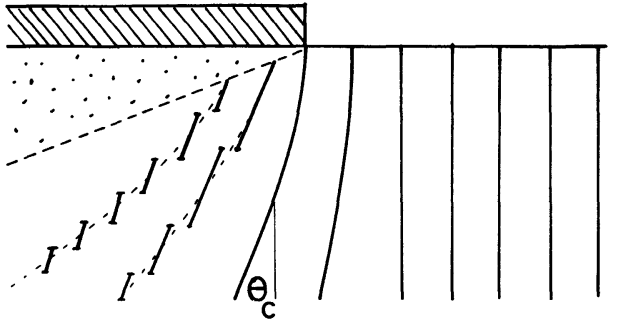

Fig. 5. - Distortion of a CDW near a contact deposited along the sample. Above a bending angle $\theta_{c}$ defects appear and the CDW should be broken in the contact vicinity.

sample cross-section or around the crystal ends, the threshold voltage is determined essentially by the nucleation energy of one dislocation loop in a perfect CDW. This case seems to be easily realizable only on blue bronze crystals which are large enough. On the other hand, for needle-like crystals of $\mathrm{NbSe}_{3}$, the contacts are deposited on the surface parallel to the chain axis, thus the equipotentials are locally perpendicular to the equiphases of undistorted CDW. Assuming as an extreme situation that the interface tries to be at constant $\Phi=q x+\varphi$, one sees that the $\Phi$-plans tend to bend until the critical angle $\theta_{c}$ is obtained (Fig. 5). For $\theta>\theta_{c}$, defects of width $\xi_{x}$ appear by shear, with a density per unit length across the sample given by

$$
n_{\mathrm{d}}=\frac{\tan \theta-\tan \theta_{\mathrm{c}}}{\xi_{x}}
$$

For large angles the CDW breaks out and the order parameter must go to zero, matching the CDW modulation with the undistorted crystal near the interface.

This is not the only possible situation. Actually it strongly depends on the nature of the, here, metalsemimetal interface. More coherence can be maintained in the volume if the CDW breaks in the interface vicinity.

Secondly, under an electric field, blocking of the phase at the contact border allows to reach a highly strained configuration in the neighbourhood, even in low fields. The «elastic limit » is easily reached and CDW defects are nucleated by shear even in very low fields.

Assuming that CDW defects are present in zero field for this type of contact geometry, no barrier opposes the nucleation of new defects on CDW motion, for the critical strains are reached progressively as the phase front goes forward. The only barrier to depinning comes from lattice friction on defect motion, operating only if $\xi \sim a$, or from pinning of dislocations by impurities. One can roughly estimate the work produced by the PeachKoehler force (Eq. (3.7)) $F=\sigma \lambda L$ to depin a 
length $L$ of dislocation to be $W \sim \sigma(\lambda L \xi)$. Using (4.6) yields

$$
V_{T}=\frac{2 W}{n_{\mathrm{s}} e(\xi L \lambda)}
$$

If $W \sim 10^{-1} \mathrm{eV}$ is the impurity pinning potential and $\xi \sim 10 \lambda, L \sim 100 \lambda, n_{s}(\xi L \lambda) \sim 2 \times 10^{3}$ and the order of magnitude of $V_{T}$ is $0.1 \mathrm{mV}$, which has been found experimentally in the best cases [39].

\subsection{CROSSOVER BETWEEN IMPURITY AND CONTACT} PINNING. - Let us discuss the competition between impurity and contact depinning as the distance $L$ between contacts is varied. When the impurity critical field $E_{T}^{\mathrm{i}}$-which is independent of sample size, provided the latter is larger than the Fukuyama-LeeRice (F.L.R.) pinning length- is reached, the CDW takes a configuration given by equation (4.2) (see Fig. 4). If $E_{T}^{\mathrm{i}} L>V_{T}$ the critical voltage for contact depinning (Eq. (4.5)), the CDW starts sliding and the observed threshold can be safely attributed to impurity pinning in the volume. If on the contrary $E_{T}^{\mathrm{i}} L<V_{T}$ the CDW becomes highly polarized but the real threshold occurs only at a higher value $E_{T}^{\mathrm{c}}$ determined by the contacts. The crossover between the two regimes $E_{T}=E_{T}^{\mathrm{i}}$ (independent of $L$ ) and $E_{T}=E_{T}^{\mathrm{c}}=V_{T} / L$ occurs at a crossover length

$$
L_{\mathrm{c}}=\frac{V_{T}}{E_{T}^{\mathrm{i}}}
$$

thus with $E_{T}^{\mathrm{i}}=100 \mathrm{mV} \cdot \mathrm{cm}^{-1}$ and $V_{T} \sim 1-10 \mathrm{mV}$, $L_{\mathrm{c}}$ ranges from $100 \mu \mathrm{m}$ to $1 \mathrm{~mm}$, which is again in agreement with experiments. In this picture $L_{\mathrm{c}}$ is absolutely unrelated to the F.L.R. pinning length. The expected variation of $E_{T}$ with $L$ is represented in figure 6.

A consequence of this picture is the existence of an intermediate regime for short samples, $E_{t}^{\mathrm{i}}<E<E_{T}^{\mathrm{c}}$, for which high polarizations can be detected as well as precursor effects to depinning such as broad band noise.

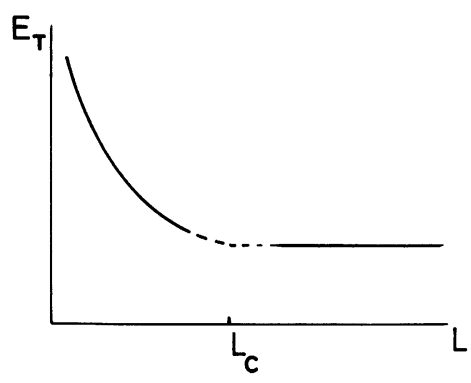

Fig. 6. - Variation with the sample length $L$ of the threshold field. Impurity pinning dominates for $L>L_{\mathrm{c}}$ while contact pinning prevails for $L<L_{\mathrm{c}}$ with $E_{T} \sim L^{-1}$.
4.3 SURFACE PINNING. - As proposed by Gill [9, 40] and one of us [22] and discussed more lengthily in reference [23], surfaces parallel to the chain axis can pin the CDW if they are rough. This can be understood imagining steps on the surface, each step involving frontal pinning as discussed above (see Fig. 7a). Describing such pinning effects by the condition $\varphi=0$ for $y=0$ and $y=L^{\prime}$ (Fig. 7b) an equation similar to (4.1) leads to the solution

$$
\varphi(y)=\frac{n_{\mathrm{s}} e E}{2 q K_{\perp}} y\left(L^{\prime}-y\right)
$$

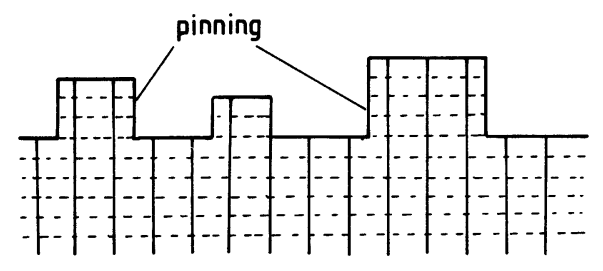

a)

b)

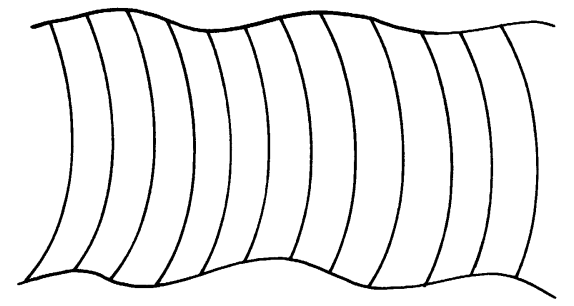

Fig. 7. - a) Possible mechanism for surface pinning on a rough surface; b) Shear configuration of the CDW.

which is very similar to (4.2). The maximal shear strain is

$$
e_{\mathrm{Max}}=\frac{n_{\mathrm{s}} e E L^{\prime}}{4 q^{2} K_{\perp}}
$$

and must be compared to the critical strain (3.12) for loop nucleation by shear. This leads to a «plastic» depinning from the sample surfaces at a critical field inversely proportional to the transverse size $L^{\prime}$ of the sample. This has been recently verified by Gill [40]. Just as for contact pinning, a crossover length $L_{\mathrm{c}}^{\prime}$ can be found, and one gets from (3.12), (4.9) and (4.11)

$$
\frac{L_{\mathrm{c}}^{\prime}}{L_{\mathrm{c}}}=\frac{\xi_{\perp}}{\xi_{x}}
$$

which gives an order of magnitude of $10 \mu \mathrm{m}$ for $L_{\mathrm{c}}^{\prime}$. 
4.4 REMANENT DEFORMATIONS OF MOVING CDW's. - All the preceding considerations applied for fields smaller than threshold. Nevertheless coherent deformations described by a parabolic profile like (4.2) should subsist above $E_{T}$. Indeed when depinning is dominated by the contacts, the maximal phase deformation, obtained at $x=L / 2$ is $\Delta \varphi=$ $\left(n_{\mathrm{s}} e / 8 q K_{x}\right) E_{T} L^{2}$ and is much larger than $2 \pi$ if $L \gg \xi_{x}^{\mathrm{f}}$ given by (4.3). One gets easily the relation

$$
\xi_{x}^{\mathrm{f}} \simeq \sqrt{8 \pi L \xi_{x}}
$$

showing that $(\Delta \varphi / 2 \pi) \sim\left(L / \xi_{x}^{\mathrm{f}}\right)^{2} \sim L / 8 \pi \xi_{x} \gg 1$.

When the CDW starts sliding, the phase at $x=0$ and $x=L$ slips discontinuously of $2 \pi-$ this causes the narrow band noise in the model of Ong and Maki [31] — but the deformation can hardily relax to zero. A steady state is obtained, characterized by a parabolic deformation, possibly weaker that (4.2). This is true only if contact pinning is stronger than bulk impurity pinning.

Such deformations have been actually found in o- $\mathrm{TaS}_{3}$ [41] and detected by resistance variations, themselves interpreted by a linear relationship between the gap $\Delta$ and the phase gradient $\partial \varphi / \partial x$. This last quantity can be interpreted as a local variation $\delta q_{\|}$of the modulation wavevector, thus of the Fermi vector, if $\delta q_{\|}$is much smaller than the ThomasFermi wavevector $k_{0}$, which is true in the semiconducting range. In a one-dimensional tight-binding band $\varepsilon_{k}=2 t \cos k a$, the gap

$$
\Delta \sim 2 \varepsilon_{\mathrm{F}} \exp \left(-1 / \lambda_{\mathrm{ep}}\right), \quad \lambda_{\mathrm{ep}}=g_{q}^{2} N\left(\varepsilon_{\mathrm{F}}\right) /\left(\hbar \omega_{q}\right)^{2},
$$

varies essentially with the density of states $N\left(\varepsilon_{\mathrm{F}}\right)$, i.e.

$$
\frac{\delta \Delta}{\Delta} \sim-\left(\frac{\delta k_{\mathrm{F}}}{k_{\mathrm{F}}}\right) \frac{k_{\mathrm{F}} a}{\tan k_{\mathrm{F}} a}\left(\frac{1}{\lambda_{\mathrm{ep}}}\right) .
$$

For a nearly one-quarter filled band, $\tan \left(k_{\mathrm{F}} a\right)=1$ and $|\delta \Delta / \Delta| \sim\left(1 / \lambda_{\mathrm{ep}}\right)(\delta q / q)$ with $\lambda_{\mathrm{ep}}=0.25$ and $(\delta q / q)=e_{x x}^{\mathrm{c}} \sim 10^{-2}$ as given by (3.12) one gets a maximal value of $\delta \Delta / \Delta \sim 0.04$ which is coherent with that found in reference [41].

\section{Conclusion.}

Considering the CDW as a three-dimensional electronic «solid», we have developed a theory of elastic and plastic deformations based on the rigidity of the modulation. Such a theory is similar to that of uniaxially strained crystals and still simpler because the position of the CDW is defined by a scalar parameter, the phase $\varphi$. In this view a CDW can be thought of a solid in the generalized meaning given by Anderson [20].

The combined action of an external electric field coupling to the phase and pinning centres (impuri- ties, surfaces or interfaces) produces strains and stresses, described by an anisotropic elastic modulus. Shear of the CDW is actually nearly one hundred times easier than compression (see also Gill [9]).

Taking advantage of the comparison with mechanical properties of solids, the necessity of a finite field to depin the CDW is obviously the clearest manifestation of solid friction. In that sense the depinning process which involves the creation and multiplication of CDW dislocations corresponds to an elastic limit of the $\mathrm{CDW}$ solid. Here the most likely mechanism for defect nucleation is strain accumulation on pinning barriers, with or without the help of thermal activation.

Contrarily to the case of a strongly commensurate CDW where flow would occur essentially by defect motion [21], in incommensurate CDW much evidence exists now for a more or less coherent motion of the CDW itself $[10,11]$. In such a context plastic flow, i.e. defect motion, occurs at strong pinning barriers, allowing the CDW current to vary strongly in space. This occurs necessarily at contacts but also on rough surfaces and possibly on strong volume pinning centres. More generally defect motion adds a new channel to CDW transport, as stressed by Lee, Rice [25] and allows an inhomogeneous distribution of velocities like that observed in blue bronzes. The contact geometry is very important in this respect, the most perturbing configurations allowing the weakest depinning fields.

Such a picture is extremely useful for obtaining correct orders of magnitude of low temperature depinning voltages at contacts. Neglecting amplitude variations allows to write linear equations but prevents one from obtaining an accurate variation of $E_{T}$ with sample length, as found numerically [36]. Nevertheless our approach accounts for the main features of the dependance of threshold fields with sample dimension, i.e. $E_{T} \sim L^{-1}$ for small sizes. This dependance is another characteristic of rigidity, i.e. long-range elastic forces, and breaks down at a crossover length which depends on the strength of impurity pinning in the volume. Depinning at boundaries is an essentially athermal effect driven by the nucleation under stress of small dislocation loops. However, thermal fluctuations could help such a mechanism if the CDW is soft enough or at higher temperatures. More generally we were able to estimate an « elastic limit » $e_{\mathrm{c}}$ in both compression or shear, which is essential for depinning from strong pinning centres. This limit can be reached locally, allowing defects to nucleate and then propagate, rendering easier the global depinning of CDW's. Such mechanisms are indeed extremely similar to what is encountered in «mechanical » elasticity of crystals. However, the fact that defect formation should be crucial for depinning does not preclude a coherent sliding of CDW's above threshold. But it 
allows for inhomogeneous CDW current as seen in big samples.

As has been qualitatively discussed [23] CDW defects can increase dramatically all metastability effects related to the remanence of deformations. Nevertheless, it is still difficult to separate the defect contribution from that coming from the disordered configuration of CDW's in weak pinning. Work is in progress to investigate the role of defects in strong impurity pinning.

We have not attempted here to describe the role of defects in stationnary CDW current. The plastic flow on strong barriers poses a difficult problem of rheology which could perhaps be solved using an hydrodynamic approach for the defects themselves. More information would be thus needed about defect interactions. Such a picture could lead to a simple qualitative explanation to the so-called « switching » phenomena : dynamical friction of solid origin is generally weaker than static friction, allowing a fall of resistance at the onset of motion or even a negative resistance regime. Instabilities observed in blue bronzes just below and above threshold could have the same origin and manifest a «stickslip friction " [43].

Analogous considerations could apply to more « exotic » electronic crystals such as Wigner crystals [44] in two dimensions. On the other hand, a CDW is a highly quantum crystal (see the Introduction) and its defects should be more likely compared for instance to those of solid helium [45]. They also bear an obvious resemblance to defects in type II superconductors and superfluids or liquid crystals.

\section{Acknowledgments.}

One of the authors (D. F.) wishes to thank Prof. L. Kubin for stimulating correspondance and Prof. S. Barisic, Drs A. Bjelis and P. Monceau for many discussions held at the International Center for Theoretical Physics (Trieste).

\section{Appendix.}

We have treated the CDW as an elastic medium and neglected amplitude variations which should occur for large strains. Let us estimate the corrections thus induced in strong pinning. The total free energy writes

$$
\begin{aligned}
F=F_{0} & \int \mathrm{d} x\left[\frac{1}{2} \xi^{2}\left|\frac{\mathrm{d} \psi}{\mathrm{d} x}\right|^{2}-t|\psi|^{2}+\frac{1}{2}|\psi|^{4}\right]- \\
& -\sum_{i} \operatorname{Re} \int \mathrm{d} x \rho_{1} v\left(x-x_{i}\right) \psi(x) \mathrm{e}^{-i \varphi_{i}}
\end{aligned}
$$

where $|\psi|=\Delta / \Delta_{0}$ (see Eq. (2.3) and (2.4))

Let us consider a simple case of strong pinning, i.e. the phase interpolating between values $\varphi_{1}$ and $\varphi_{2}$ at $x=0$ and 1 respectively. Rather than solving the nonlinear equation resulting from $\partial F / \partial \psi=0$, one can simply remark that

$$
\left|\frac{\mathrm{d} \psi}{\mathrm{d} x}\right|^{2}=\left(\frac{\mathrm{d}|\psi|}{\mathrm{d} x}\right)^{2}+|\psi|^{2}\left(\frac{\mathrm{d} \varphi}{\mathrm{d} x}\right)^{2} .
$$

Therefore :

(i) a change of $|\psi|$ slightly modifies the elastic constant for phase deformations ;

(ii) the presence of a longitudinal strain $e_{x x}$ leads to a reduction of $|\psi|$ to second order in $e_{x x}$. In the present situation $e_{x x}=(1 / q) \mathrm{d} \varphi / \mathrm{d} x \approx\left(\varphi_{2}-\varphi_{1}\right) / l$ and $v\left(x-x_{i}\right) \approx 0(i=1,2)$ between pinning points, thus (A.1) yields

$$
|\psi| \sim t^{1 / 2}\left[1-\frac{\xi^{2} q^{2}}{4 t} e_{x x}^{2}\right] .
$$

On the contrary, in the vicinity of pinning points $\mathrm{d} \varphi / \mathrm{d} x \approx 0$ and $F$ writes

$$
\begin{aligned}
F=F_{0} \int \mathrm{d} x\left[\frac{1}{2}|\psi|^{4}-t|\psi|^{2}\right]- \\
-\int \mathrm{d} x \rho_{1} v\left(x-x_{i}\right)|\psi|
\end{aligned}
$$

Minimizing with respect to $|\psi|$ yields

$$
|\psi| \sim t^{1 / 2}\left[1+\frac{\rho_{1}|v(0)|}{4 F_{0} t^{3 / 2}}\right]
$$

As mentioned by Lee and Rice [25] the order parameter is enhanced near a strong impurity. The matching between the two solutions, given by (A.3) and (A.5) and sketched in figure 8 needs a selfconsistent calculation. It most probably occurs on a length of order $\xi$, which justifies our estimation if $l \gg \xi$.

As a conclusion, a variation of the CDW wavevector along the chain direction affects the order parameter in two ways. First, the Ginzburg-Landau expansion leads to a second order effect expressed by (A.3). With $t=1$ at $T=0, e_{x x} \sim e_{x x}^{\mathrm{c}}=10^{-2}$, the maximal correction is of the order of some percents. Secondly, as pointed out in section 4.4 , with an order of commensurability equal to 4 , a first order effect arises from the density of states variation. Our estimations show that the two effects are comparable in magnitude and may add or compete together.

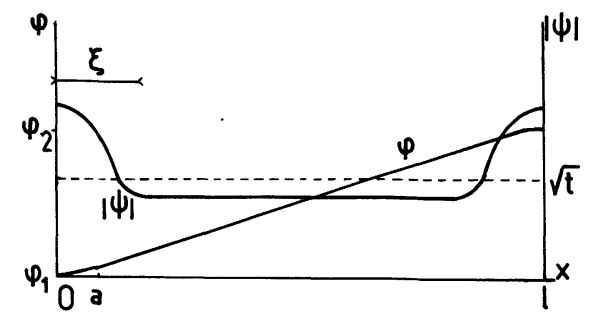

Fig. 8. - Variation of the CDW amplitude $|\psi|$ for the phase interpolating between two strong pinning points in one dimension. 


\section{References}

[1] Monceau, P., Ong, N. P., Portis, A. M., Meerschaut, A. and Rouxel, J., Phys. Rev. Lett. 37 (1976) 602.

[2] For reviews see

a) Electronic Properties of Inorganic Quasi-OneDimensional Compounds, Ed. Monceau P. (Reidel, Dordrecht) 1985 ;

b) Crystal Chemistry and Properties of Materials with Quasi-One-Dimensional Structures, Ed. Rouxel J. (Reidel, Dordrecht) 1986 ;

c) Grüner, G. and Zettl, A., Phys. Rep. 119 (1985) 117

d) Gill, J. C., Contemp. Phys. 27 (1986) 37.

[3] Lacoe, R. C., Schulz, H. J., JÉrome, D., Bechgaard, K. and Johanssen, I., Phys. Rev. Lett. 55 (1985) 2351.

[4] LeE, P. A., Rice, T. M. and Anderson, P. W., Solid State Commun. 14 (1974) 703.

[5] Charge Density Waves in Solids, Ed. Hutiray, G. and Solyom, J., Lecture Notes Phys., vol. 217 (Springer, Berlin) 1985.

[6] Fleming, R. M., Dunn, R. G. and Schneemeyer, L. F., Phys. Rev. B 31 (1985) 4099.

[7] Tamegai, T., Tsutsumi, K., Kagoshima, S., KanaI, Y., TOMOZawa, H., TANI, M., NoGANI, Y. and SATo, M., Solid State Commun. 56 (1985) 13.

[8] Brown, S. E., Mihaly, L. and Grüner, G., Solid State Cómmun. 58 (1986) 231.

[9] GiLl, J. C., in reference [5], p. 377.

[10] Ross, J. H., Wang, Z. and Slichter, C. P., Phys. Rev. Lett. 56 (1986) 663.

[11] Segransan, P., Janossy, A., Berthier, C., MarCus, J. and Butaud, P., Phys. Rev. Lett. 56 (1986) 1854.

[12] Peierls, R. E., Quantum Theory of Solids (Oxford University Press) 1955, p. 108.

[13] Bardeen, J., Phys. Rev. Lett. 45 (1980) 1978 ;

BARDEEN, J. and TUCKER, J. R., in reference [5], p. 155.

[14] Sneddon, L., Cross, M. C. and Fisher, D. S., Phys. Rev. Lett. 49 (1982) 292.

[15] Klemm, R. A. and Schrieffer, J. R., Phys. Rev. Lett. 51 (1983) 47 ;

Fisher, D. S., Phys. Rev. B 31 (1985) 1396.

[16] Thorne, R. E., Tucker, J. R. and Bardeen, J., Phys. Rev. Lett. 58 (1987) 828.

[17] TÜttö, I. and Zawadowski, A., Phys. Rev. B 32 (1985) 2449

[18] BARDEEN, J., preprint.

[19] Aubry, S., in Bifurcation Phenomena in Mathematical Physics and Related Topics, Eds Bardos, C. and Bessis, O. (D. Reidel) 1980, p. 163.

[20] Anderson, P. W., Basic Notions of Condensed Matter Physics (Benjamin Cummings ed.) 1984, p. 159.

[21] Dumas, J. and Feinberg, D., Europhys. Lett. 2 (1986) 555

[22] FRIEDEL, J., in NATO ASI on Low Dimensional Conductors and Superconductors, Eds Caron, L. and Jérôme, D., Magog (Canada), August 1986 (Plenum, to be published).

[23] Feinberg, D. and Friedel, J., in Low Dimensional Electronic Properties of Molybdenum Bronzes and Oxides, Ed. Schlenker, C. (Reidel, Dordrecht) to be published.

[24] MacMillan, W. L., Phys. Rev. B 12 (1975) 2042.

[25] Lee, P. A. and Rice, T. M., Phys. Rev. B 19 (1979) 3970.

[26] Fukuyama, H. and Takayama, H., in reference [2] a) Vol. I, p. 41

[27] Nakane, Y. and Takada, S., J. Phys. Soc. Jpn 56 (1985) 977.

[28] Barisic, S., Mol. Cryst. Liq. Cryst. 119 (1985) 413 and same reference as [22].

[29] PutTerman, S. J., Superfluid Hydrodynamics (North Holland, Amsterdam) 1974.

[30] MAKI, K., Physica 143B (1986) 59.

[31] ONG, N. P. and MAKI, K., Phys. Rev. B 32 (1985) 6582.

[32] Friedel, J., Dislocations (Pergamon Press, Oxford) 1964.

[33] Shockley, W., L'Etat Solide, Proceedings of the Neuvième Conseil de Physique Solvay (R. Stoops, Bruxelles) 1952.

[34] Feynman, R. P., in Progress in Low Temperature Physics, Ed. Gorter, C. J., Vol. I (North Holland, Amsterdam) 1955.

[35] Villain, J., Z. Phys. B 54 (1984) 139.

[36] Batistic, I., Bjelis, A. and Gor'Kov, L. P., J. Phys. France 45 (1984) 1049.

[37] Arbaoui, A., Thesis, Université de Grenoble (1985).

[38] Mihaly, G., Hutiray, Gy. and Mihaly, L., Phys. Rev. B 28 (1983) 4896.

[39] Monceau, P., Renard, M., Richard, J. and Saint-Lager, M. C., Physica 143B (1986) 64 ;

SaINT-Lager, M. C., Thesis, Université de Grenoble (1983).

[40] Yetman, P. J. and Gill, J. C., to be published.

[41] Brown, S. E., Mihaly, L. and Grüner, G., Solid State Commun. 58 (1986) 231.

[42] Janossy, A., Mihaly, G. and Kriza, G., Solid State Commun. 51 (1984) 63.

[43] Dumas, J., Arbaoui, A., Guyot, H., Marcus, J. and SChlenker, C., Phys. Rev. B 30 (1984) 2249 ; see also

Dumas, J. and Schlenker, C., in reference [2] b) p. 135.

[44] Rosenbaum, T. F., Field, S. B., Nelson, D. A. and Littlewood, P. B., Phys. Rev. Lett. 54 (1985) 241 ;

Field, S. B., Reich, D. H., Shivaram, B. S., Rosenbaum, T. F., Nelson, D. A. and LitTlewood, P. B., Phys. Rev. B 33 (1986) 5082.

[45] NoziÈres, P., Proceedings of the 7th General Conference of the Condensed Matter Division of the European Physical Society, Pise, April 1987. 